\title{
Lorsque les valeurs changent de paradigme
}

\section{Rouven Porz}

Prof. Dr, Ethique de la médecine et formation postgraduée des médecins, Hôpital de l'lle Berne, membre de la rédaction Ethique du BMS

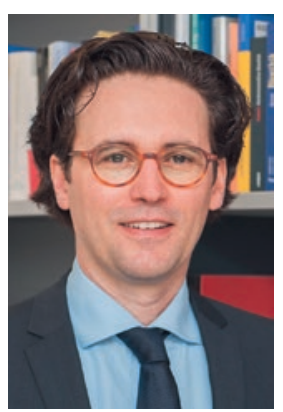

De nombreuses libertés civiles, autrement évidentes, ont été limitées ces derniers mois. Nous ne pouvons plus voyager librement, nous ne pouvons plus rencontrer toutes les personnes que nous aimerions voir, nous ne pouvons plus nous prendre dans les bras et nous embrasser lors des réunions de famille ou entre amis, participer à de grandes manifestations comme un festival de musique ou un évènement sportif était pratiquement impossible. Des restrictions, des limitations de notre liberté aux dépens de notre autodétermination. Nous n'y sommes pas habitués, c'est un fait. En Suisse, plus particulièrement, l'autodétermination est une valeur importante, peut-être même la plus importante dans la vie en communauté.

Mais la pandémie de la Covid-19 a entraîné un changement des valeurs, nous mettant face à un nouveau paradigme. Nous avons dû apprendre qu'il existe, à côté de l'autodétermination, une autre valeur essentielle qui peut être exprimée de deux manières. La première est celle de la santé publique (Public Health), tandis que la deuxième constitue la solidarité nécessaire entre les individus pour, d'un commun accord, ne pas mettre en danger cette santé publique. Nous parlons d'un conflit des valeurs perçu entre l'autodétermination et la solidarité. Ce conflit s'observe tous les jours dans les médias: d'un côté l'appel à la communauté, de l'autre côté la préservation obstinée de la «liberté» personnelle. Une contradiction pour le moins délicate. Les conflits de valeurs sont toujours épineux, car ils font référence à des idées souvent diamétralement opposées - noir ou blanc. En temps normal, nous parvenons à éviter ces confrontations avec habilité et poursuivons nos activités habituelles (autodétermination!), mais cette fois, il n'y a pas tellement moyen d'y échapper. Nous sommes en quelque sorte tous dans le même bateau (solidarité!).

Avec ce changement de paradigme émergent toutefois aussi des approches conciliantes. Il nous faut d'abord reconnaître que des personnes d'horizons différents ont des valeurs différentes, ce qui n'est pas nécessairement négatif. Deuxièmement, le blanc et le noir peuvent très bien se marier et donner un beau gris. Il est donc possible d'apprécier l'autodétermination tout en agissant de manière solidaire. Enfin, nous pouvons nous appuyer sur les propos du philosophe des Lumières probablement le plus connu, Emmanuel Kant (17241804): La «liberté» ne signifie pas avoir des droits, mais avoir des obligations [1]. Cette pensée de Kant est celle qui nous mène vers un nouveau changement de paradigme. Peut-être vivons-nous vraiment une époque où nous devrions réfléchir à nos obligations humanitaires au lieu de revendiquer uniquement nos droits individuels. Pour ma part, je vois ceci comme une invitation et une idée pour être plus conciliant.

\section{Nous devrions réfléchir à nos obligations} humanitaires au lieu de revendiquer uniquement nos droits individuels.

L'éthique médicale, ma discipline, devra aussi faire face à ce changement de paradigme. Par rapport à la valeur de la santé publique, le principe primordial de l'autonomie du patient passe actuellement quelque peu au second plan. C'est une bonne chose, car cela contraindra encore davantage notre discipline à réfléchir en profondeur à la manière dont des systèmes de valeurs en apparence contradictoires peuvent co-exister et fonctionner parallèlement. Le respect de l'autonomie du patient restera toujours une valeur extrêmement importante, mais elle ne sera peut-être plus exclusive et primordiale. La valeur de la solidarité pourrait ainsi être enfin plus présente dans les questionnements en éthique médicale. Des éléments de réflexion et des travaux préliminaires prometteurs existent déjà [2]. Peutêtre faudra-t-il même changer le nom de la discipline et qu'il est temps d'élargir le terme un peu étroit d'éthique médicale à un terme plus englobant de "Health Care Ethics».

\section{Références}

1 Immanuel Kant. Die Metaphysik der Sitten. Original 1797. [Kapitel: Einleitung in die Rechtslehre].

2 Ruud ter Meulen. Solidarity and Justice in Health and Social Care. Cambridge University Press 2017. Ou bien: Barbara Prainsack et Alena Buyx. Solidarity in Biomedicine and Beyond. Cambridge University Press 2017. 